\title{
A calcium rich natural marine-derived multi-mineral supplement has beneficial effects on lipid concentrations in postmenopausal women
}

Calcium (Ca) supplements are widely taken by postmenopausal women for the maintenance of bone health and prevention of osteoporosis. Beneficial effects on high-density lipoprotein (HDL), low-density lipoprotein (LDL) and total cholesterol (TC) have been reported following Ca supplementation either alone or with vitamin $\mathrm{D}^{(1,2)}$. Short-chain fructooligosaccharides (scFOS) have been shown in vivo to increase $\mathrm{Ca}$ absorption and thus may indirectly affect lipid status ${ }^{(3)}$. The aim of this study was to investigate the effects of a $\mathrm{Ca}$ rich natural marine-derived multi-mineral supplement (trade name Aquamin) administered alone or in combination with scFOS (trade name Nutraflora) on circulating lipid concentrations in postmenopausal women.

A total of 214 postmenopausal women (mean BMI $27 \cdot 3($ SD $4 \cdot 7) \mathrm{kg} / \mathrm{m}^{2}$ ) aged 48-75 years completed a two year double-blind placebo controlled trial. Participants were randomly assigned to daily supplements of $800 \mathrm{mg}$ of Ca $(2 \cdot 4 \mathrm{~g} \mathrm{Aquamin})(n=75), 800 \mathrm{mg}$ of Ca with $3 \mathrm{~g}$ of scFOSs $(3.2 \mathrm{~g}$ Nutraflora) (CaFOS) $(n=60)$ or maltodextrin (MD) $(n=79)$. TC, LDL, HDL and triglyceride concentrations were measured at baseline and 24 months. Per-protocol analysis using ANCOVA (with baseline measures as covariates) was conducted to assess time $\times$ treatment effects between groups, controlling for age, BMI and baseline calcium intake (mean intake $868 \mathrm{mg} \mathrm{Ca}$ /day) using least significant difference for post hoc comparisons.

A significant time $\times$ treatment effect was observed for LDL for the Ca $(P=0 \cdot 009)$ and CaFOS groups $(P=0 \cdot 01)$, see Fig. 1 . Significant time $\times$ treatment effects were found for TC for the Ca and CaFOS groups $(P=0 \cdot 02$ and $P=0 \cdot 03$ respectively), see Fig. 2. There was no significant treatment effect for the two groups for HDL, LDL:HDL and triglyceride concentrations.

Supplementation with Aquamin, a Ca rich natural marine-derived multi-mineral supplement has beneficial effects on the lipid profile of postmenopausal women over two years; a possible mechanism is Ca binding to bile acids which might lead to increased excretion of bile salts and a subsequent increase in lipolysis. Administration of Aquamin may have a role in maintaining cardiovascular health in women and further research on this area is warranted.

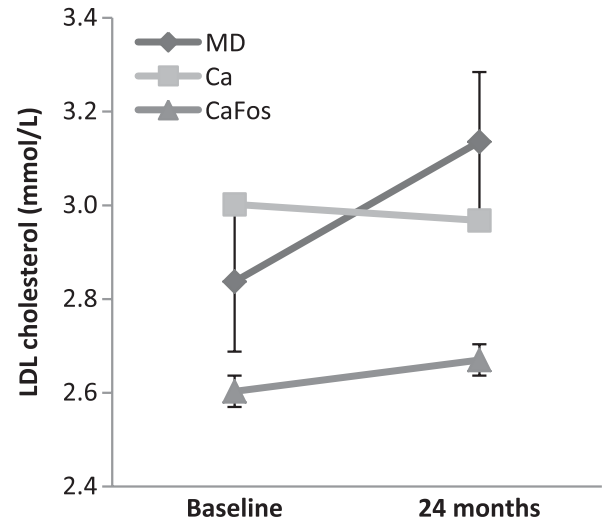

Fig. 1 Effects on LDL cholesterol over 24 months. Data are means \pm SE.

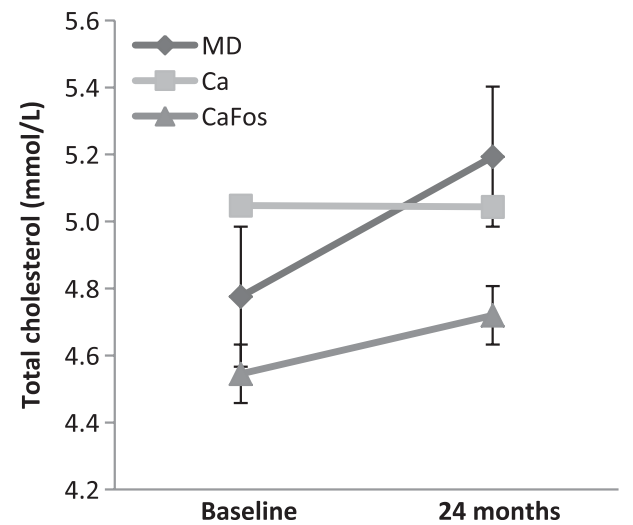

Fig. 2 Effect on Total cholesterol over 24 months. Data are means $\pm \mathrm{SE}$

This work was funded by Marigot (Cork, Ireland), Ingredion Inc. (Westchester, IL) and a PhD studentship received from the Department for Employment and Learning.

1. Reid I, Mason B, Horne A et al. (2002) Am J Med 112, 343-347.

2. Schnatz P, Jiang X, Vila-Wright S et al. (2014) Menopause (Epublication ahead of print version).

3. Morohashi T, Sano T, Ohta A et al. (1998) J Nutr 128, 1815-8. 